\title{
Paradigma na Formação Médica: Atitudes e Conhecimentos de Acadêmicos sobre Morte e Cuidados Paliativos
}

\author{
End-of-Life Paradigm in Medical Training: \\ Attitudes and Knowledge about Death and \\ Palliative Care
}

\section{PALAVRAS-CHAVE}

- Cuidado Paliativo.

- Cuidados Paliativos na Terminalidade da Vida.

- Educação Médica.

- Morte.

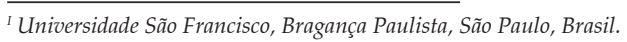

\section{RESUMO}

A Organização Mundial da Saúde (OMS) define Cuidados Paliativos como abordagem que aprimora a qualidade de vida dos pacientes e seus familiares que enfrentam problemas associados a doenças ameaçadoras de vida, por meio da prevenção e alivio do sofrimento. Recentemente no Brasil, promoveu-se a Medicina Paliativa a especialidade médica, obrigando a repensar os conceitos de educação em terminalidade da vida nas escolas brasileiras. O relacionamento pessoal com a morte parece influenciar diretamente a premissa do cuidar-mais-que-curar, sendo essencial na relação do cuidador com aquele que morre. Há estreita relação entre ansiedade e medo da morte e a atitude do estudante de Medicina perante situações de terminalidade de vida. A visão que o acadêmico tem sobre a morte poderá determinar a disponibilidade interna, valores, conceitos e preconceitos com relação à morte e ao morrer e determinar seu desempenho como profissional. Dessa forma, os programas de educação médica devem ressaltar não apenas os aspectos teóricos e técnicos em Cuidados Paliativos, mas também o clima emocional que envolve o ato médico e a finitude da vida. Objetivo: Comparar as atitudes de acadêmicos em curso de Medicina perante a morte e o processo de morrer segundo o contato teórico e/ou prático com a disciplina de Cuidados Paliativos ao longo de sua formação médica. Método: Estudo de coorte que avalia a modificação do perfil do acadêmico de Medicina com base nos questionários de Tanatofobia e Autoeficácia em Cuidados Paliativos. Resultados: A reflexão teórica diminui a ansiedade relacionada à atividade prática $(p<0,05)$. As habilidades em comunicação e multidisciplinaridade em Cuidados Paliativos são mais bem desempenhadas pelo grupo que recebe treinamento completo, teórico e prático, do que nos grupos que recebem apenas teoria ou nos que adquirem seus conhecimentos mediante aprendizado em serviço exclusivamente $(p<0,05)$. Conclusão: A disciplina Cuidados Paliativos auxilia na superação de medos relacionados à morte, reduzindo a ansiedade envolvida na prática dos cuidados de fim de vida, principalmente no quesito comunicação. Estudantes que recebem treinamento teórico e prático em Cuidados Paliativos mostram maior confiança diante de situações de terminalidade. 


\section{KEYWORDS}

- Palliative Care.

- Hospice Care.

- Education.

- Medical.

- Death.

\begin{abstract}
Background: The World Health Organization (WHO) defines Palliative Care as an approach which, by preventing and alleviating suffering, improves the quality of life of patients and their families who face problems associated with life-threatening illnesses. In Brazil, Palliative Medicine has recently been promoted to the category of medical specialty, obliging Brazilian medical schools to review the educational concepts associated to end-of-life care. The personal relationship with death seems to directly influence the premise of care-more-than-healing, as an essential element of the caregiver's relationship with the terminal patient. There is a close relationship between anxiety and fear of death and the medical student's attitude in the face of end-of-life situations. The scholar's view of death may determine their inner disposition, values, concepts, and prejudices regarding death and dying and determine their performance as a professional. Thus, medical education programs must emphasize not only the theoretical-technical aspects in Palliative Care, but also the emotional climate that involves the medics' attitudes and actions in end-of-life situations. Objective: To compare the attitudes of medical students when faced with death and the process of dying according to their theoretical and/or practical training in Palliative Care. Method: A cohort study evaluating how the profile of medical students has changed based on questionnaires on Fear of Death and Self-Efficacy in Palliative Care. Results: Theoretical reflection reduces anxiety related to practical activity $(p<0.05)$. Communication and multidisciplinary skills in Palliative Care are performed far better by those who receive complete theoretical and practical training than those who are trained only in theoretical terms or those who rely exclusively on in-service learning $(p<0.05)$. Conclusion: The Palliative Care discipline helps students overcome fears related to death, thus reducing their anxiety when practicing end-of-life care, especially in terms of communication. Students who receive this theoretical and practical training. Students who receive theoretical and practical training in Palliative Care show greater confidence when faced with end-of-life situations.
\end{abstract}

Recebido em: 27/10/17

Aceito em: 7/11/17

\section{INTRODUÇÃO}

O conceito de Cuidado Paliativo (CP) tem origem no movimento hospice, idealizado por Cecily Saunders em 1967 na Inglaterra, e descreve a filosofia do cuidado da pessoa em processo de finitude com o objetivo de aliviar o sofrimento físico, psicológico, social e espiritual com o propósito final de cuidar e não de curar ${ }^{1}$.

Em 2002, a Organização Mundial da Saúde (OMS) define $\mathrm{CP}$ como abordagem que aprimora a qualidade de vida dos pacientes e seus familiares que enfrentam problemas associados com doenças ameaçadoras de vida por meio da prevenção e alívio do sofrimento. Esta abordagem preconiza a identificação precoce, avaliação correta e tratamento da dor e outros problemas de ordem física, psicossocial e espiritual ${ }^{2}$. A qualidade de vida do paciente é o objetivo, desde o momento do diagnóstico da doença, que poderá ser crônica, degenerativa e/ou incurável, e não o cuidar dirigido ao leito de morte apenas. Desta forma, a abordagem poderia ser encarada como cuidados continuados.
O Código de Ética Médica (2009) veda ao médico abreviar a vida do paciente, ainda que a pedido deste ou de seu representante legal, sendo que, nos casos de doença incurável e terminal, deverá o "médico oferecer todos os $\mathrm{CP}$ disponíveis, sem empreender ações diagnósticas ou terapêuticas inúteis ou obstinadas, sempre considerando a vontade expressa do paciente ou, na sua impossibilidade, a de seu representante legal”3 (p. 90).

No Brasil, somente em 2010 a Medicina Paliativa foi considerada área de atuação médica com formação específica. Este constitui um grande passo para os CP no País, pois, dessa forma, consegue se afirmar oficialmente, facilitando a formação de políticas públicas de saúde voltadas para a área, assim como a maior preocupação de introduzir definitivamente a educação em morte e terminalidade da vida em currículos das escolas médicas. Citando Maria Goretti Sales: "É preciso educar para paliar, legitimar a prática, envolver profissionais" 4 (p. 6).

Em realidade muito distinta da realidade brasileira, na metade da década de 1970, 6\% das escolas de Medicina dos Estados Unidos ofereciam curso sobre a morte e o morrer ${ }^{5}$. Já na década 
de 1990, 30\% das escolas médicas daquele país ofereciam formação dedicada aos cuidados em terminalidade da vida, e quase todos os programas incluíam algum aspecto desse tópico como parte de alguma disciplina ${ }^{6}$. O Liaison Committee on Medical Education (LCME) determinou que todos os currículos americanos devem incluir conteúdo de CP nos exames de licenciamento, estimulando o aumento no ensino desta área de tal forma que, em 2002, 97\% dos estudantes de Medicina foram expostos ao ensino de $\mathrm{CP}$ e várias escolas ofereceram tópicos curriculares neste tema. A partir de 2007, o Medical School Palliative Care Education Project, dirigido pelo End of Life/Palliative Education Resource Center, desenvolveu rotinas clínicas orientadas nos últimos dois anos das escolas de Medicina ${ }^{7}$.

Em 2012, um estudo analisou o ensino em terminalidade da vida nas escolas médicas brasileiras e verificou que, dos $50 \%$ participantes, $35,1 \%$ ofereciam disciplina, curso ou eletivo em CP, sendo obrigatório em apenas 33,9\% do total destas escolas ${ }^{8}$. Sabe-se que o tema é abordado de maneira não contínua, não obrigatória e em disciplinas ligadas ao tema da morte, dor, ou relacionado à área de Oncologia, ou seja, não se trata da questão de maneira sistemática como se trata, por exemplo, a anatomia ou a propedêutica9. Um estudo recente, que compara a educação médica em $\mathrm{CP}$, observou preocupação com o tema em diversos países, mas com pouca resolutividade quanto a como solucionar este problema ${ }^{10}$. A partir desse estudo, foram traçadas estratégias individualizadas, em poucas das muitas universidades estudadas, com resultados positivos, mas divulgados em escala mínima. Também foi atestado que a dificuldade em atingir o método ideal de ensino se relaciona à falta de profissionais capacitados para ensinar e assumir a disciplina dentro de serviços médicos por ser este um tema muito novo na medicina em geral ${ }^{10}$.

O estudo Support ${ }^{11}$, realizado nos Estados Unidos em 1995, avaliou indicadores de qualidade no cuidado recebido por mais de 4 mil pacientes terminais em hospitais universitários e revelou grande deficiência dos médicos quanto ao conhecimento do desejo de ressuscitação cardiopulmonar dos pacientes, tempo de permanência em UTI e ventilação mecânica e controle de dor. Isto demonstra a necessidade da educação para clínicos gerais em medicina paliativa, uma vez que $70 \%$ de 897 sujeitos de pesquisa não consideravam ter preparo suficiente para o manejo dos cuidados em terminalidade da vida. Em 2014, um estudo belga com médicos generalistas demonstrou que, em sua maioria, estes profissionais consideraram a educação em CP recebida durante a graduação como insuficiente e excessivamente focada em temas bioéticos, excluindo importantes aspectos, como farmacologia e multidisciplinaridade. Também cita a importância de experimentar situações reais ligadas à teoria ${ }^{12}$.
A esperança de que existam profissionais mais sensíveis às necessidades da população e do indivíduo, que visem atender à pessoa doente mais que à doença e que se mobilizem numa assistência mais humanizada, se concentra na academia, que deve estimular o ensino centrado na pessoa do paciente e na família. A aquisição destes valores poderá ser proporcionada por currículos que privilegiam as competências inerentes a cada curso da área da saúde, assim como a abordagem multiprofissional, a comunicação e a bioética enquanto conteúdos imprescindíveis, cobrindo todas as etapas do ciclo vital, inclusive a terminalidade e a morte ${ }^{13}$.

Um fator que parece influenciar diretamente a premissa do cuidar-mais-que-curar é o relacionamento pessoal com a morte, sendo essencial na relação com aquele que morre. A visão que o estudante tem sobre a morte poderá determinar a disponibilidade interna, valores, conceitos e preconceitos com relação à morte e ao morrer. Um estudo anterior pôde estabelecer estreita relação entre ansiedade e medo da morte e atitude do aluno perante situações de terminalidade de vida ${ }^{14}$. Dessa forma, os programas de educação médica continuada devem ressaltar não apenas os aspectos teóricos e técnicos em $\mathrm{CP}$, mas também o clima emocional que envolve o ato médico e a finitude da vida ${ }^{15}$.

É neste contexto que se faz necessária a inclusão de disciplina voltada ao estudo do processo de morte e de morrer, tratando, além do conhecimento do manejo de fármacos e nutrição, avaliação e controle da dor e outros sintomas, da habilidade em comunicação e da questão da terminalidade e seus aspectos éticos e legais associados à clínica diária da equipe de saúde.

Adotando a hipótese de que a educação em paliativismo durante a formação médica, através de disciplina com foco primário, leva ao melhor desempenho dos futuros médicos perante pacientes em situação de terminalidade de vida, este trabalho objetiva comparar as atitudes de acadêmicos em curso de Medicina perante a morte e o processo de morrer segundo o contato teórico e/ou prático com a disciplina de $\mathrm{CP}$ ao longo de sua formação médica.

\section{MÉTODO}

\section{Adequação do projeto}

O estudo foi realizado na Universidade São Francisco, Campus de Bragança Paulista (SP), e os sujeitos são os acadêmicos do curso de Medicina. Foi conduzido com auxílio do Cremesp por meio do programa de pesquisa em bioética. Obedeceu às etapas previstas pelo Comitê de Ética em Pesquisa da Universidade São Francisco (CAAE: 0177.0.142.000-11) e às normativas Nacionais (Conep - Conselho Nacional de Saúde do Ministério da Saúde do Brasil). Todos os participantes selecionados para o estudo assinaram o Termo de Consentimento Livre e Esclarecido. 


\section{Descrição do contexto da disciplina}

Esse estudo só foi possível graças à modificação da estrutura curricular do curso de Medicina da Universidade São Francisco durante o período em que foi aplicado (2011 a 2016). À mesma época, havia discentes em fase final do internato do currículo em extinção, em que não havia a disciplina, e discentes ingressantes no novo currículo, no qual foi inserida a disciplina de $\mathrm{CP}$, e que foram acompanhados até o internato. Trata-se de estudo de coorte feito em dois momentos, M0 e M1.

A disciplina de CP é ministrada em Atualização I, no sexto semestre do curso de Medicina da Universidade São Francisco, e tem como molde o tripé da Medicina Paliativa: controle de sintomas que causam desconforto, comunicação, multidisciplinaridade e ética.

As aulas contam com a participação de profissionais da área de saúde da Nutrição, Biologia, Farmácia e Medicina; são convidados professores de áreas específicas, como Enfermagem, Psiquiatria e unidade de terapia intensiva.

São apresentados temas relacionados a medicação e técnicas para controle de sintomas e dieta do paciente paliativo; reservadas horas para reflexões de caráter legal, espiritual, social, cultural, moral e ético acerca da morte e do morrer aplicadas à atuação médica geral e aos CP em seu caráter específico; e treinamento de habilidades de comunicação com base em protocolo de dar más notícias (Class) ${ }^{16}$.

\section{Grupos}

Os grupos estão caracterizados no Quadro 1.

\section{QuAdro 1}

Caracterização dos grupos estudados e dos tempos

avaliativos do estudo. M0 - compara os grupos entre si,

num primeiro momento; M1 - avalia de forma evolutiva, ao longo do tempo, turma em progressão (coorte)

SEM TEORIA E COM PRATICA $\cdot$ Internato
$\cdot n=28$

-Alunos que estavam em curso do internato no início do estudo antes da existência da disciplina Cuidados Paliativos -Avaliação am MO

\section{SEM TEORIA E SEM PRÁTICA}

- Iniciante

- Alunos que não cursaram a disciplina Cuidados Paliativos e não tinham

experiência clínica em curso inicial

-Avaliação em M0 e M1

\section{COM TEORIA E SEM PRATICA}

-Pré-clínico

- Alunos que cursaram a disciplina Cuidados Paliativos em curso pré-clínico - Avaliação em M0 e M1

COM TEORIA E COM PRÁTICA

-Internato

$\cdot n=53$

- Alunos que cursaram a disciplina Cuidados Paliativos em curso do internato -Avaliação em M0 e M1
Questionários avaliativos e momentos de aplicação

Foram utilizados os questionários Multidimensional Fear of Death Scale (MFODS $)^{17}$ e Self-Efficacy in Palliative Care Scale $(S E P C S)^{18}$, aplicados em dois tempos distintos e analisados como estudo de coorte em dois momentos: M0 - avaliação inicial transversal (executada no período de agosto e setembro de 2011); e M1 - avaliação de coorte (executada em dezembro de 2012), conforme o Quadro 1.

O Multidimensional Fear of Death Scale avalia o medo da morte por meio de 42 questões curtas sobre situações relacionadas à morte, nas quais a resposta varia numa escala de 1 a 5 , onde 1 é "concordo fortemente" e 5 é "discordo fortemente". Ao final do teste, estas questões são divididas em oito grupos de medo da morte: medo do processo de morrer (1), dos mortos (2), de ser destruído (3), medo por aqueles importantes para você (4), do desconhecido (5), da morte consciente (6), pelo corpo depois da morte (7) e da morte prematura (8). Por fim, observa-se em quais aspectos a morte gera maior ansiedade ao indivíduo.

O Self-Efficacy in Palliative Care Scale apresenta 23 sentenças relacionadas a assuntos e experiências que poderão ser apresentados em unidade de tratamento paliativo relacionadas a comunicação, técnica específica e trabalho em equipe multidisciplinar. As respostas variam em uma linha traçada entre os extremos "muito ansioso" e "muito confiante". O indivíduo julga onde se encontra em cada situação proposta. As respostas são divididas em quatro espaços para avaliar o grau de ansiedade do indivíduo.

\section{Critérios de exclusão}

Preenchimento equivocado dos questionários de forma a inviabilizar sua análise e já ter tido treinamento curricular ou extracurricular em CP.

\section{Análise estatística}

A determinação do $n$ amostral foi efetuada com uso do método de Santos ${ }^{19}$. Os resultados estatísticos foram obtidos adotando-se nível de significância de 5\% para negar a hipótese de nulidade, por meio do programa SPSS 13.0. A análise dos resultados obtidos foi feita mediante os seguintes modelos: análise descritiva, análise de tendência central e análise de variância. Para análises ao longo do tempo, quando se tratava do mesmo grupo, foi utilizado o teste de Wilcoxon (dados pareados), enquanto para a comparação entre grupos distintos foi utilizado o teste de Kruscall-Wallis. 

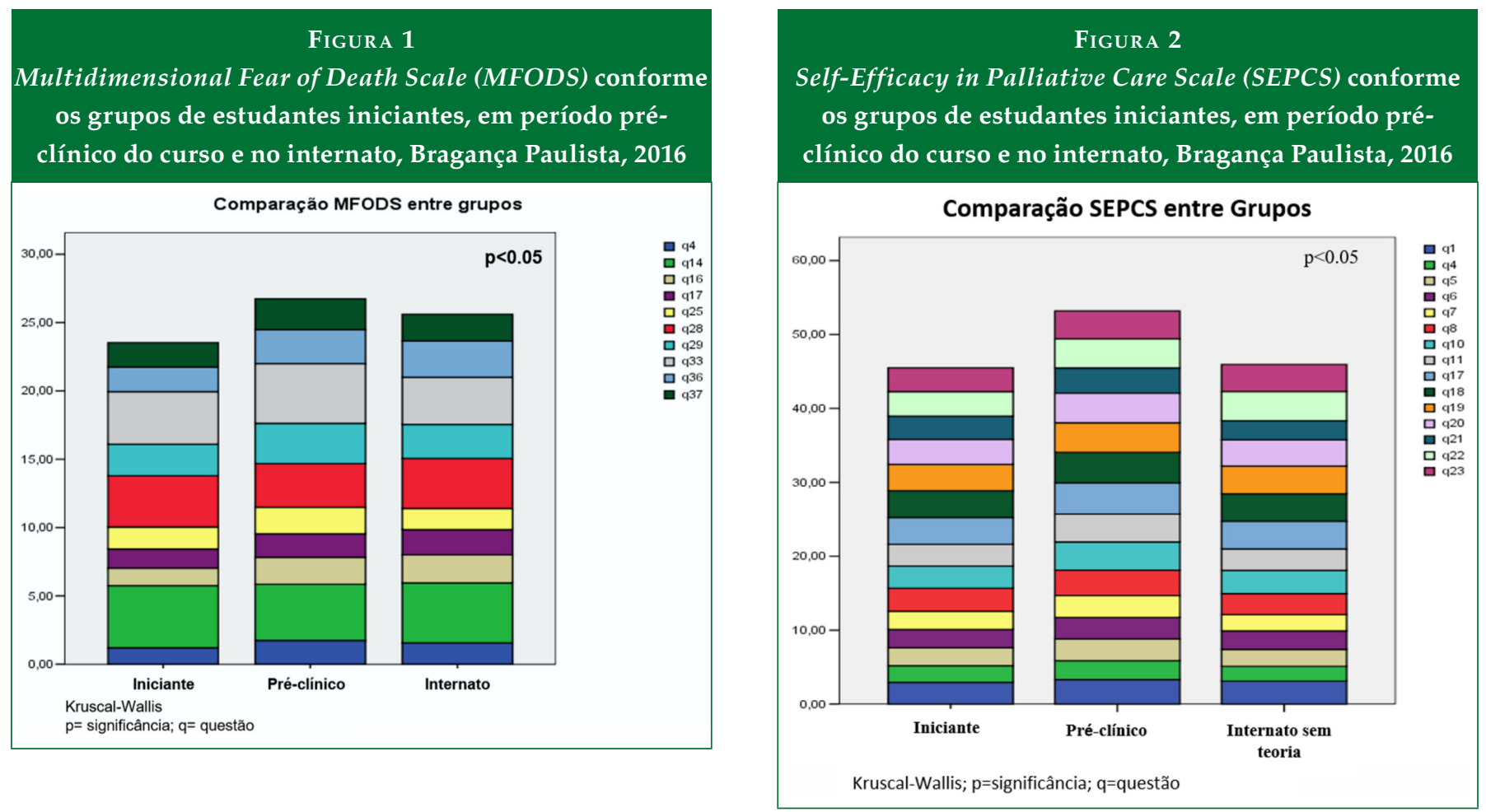

\section{Figura 3}

Pontos específicos do questionário Self-Efficacy in Palliative Care Scale (SEPCS) conforme os grupos de estudantes iniciantes, em período pré-clínico do curso e no internato, Bragança Paulista, 2016
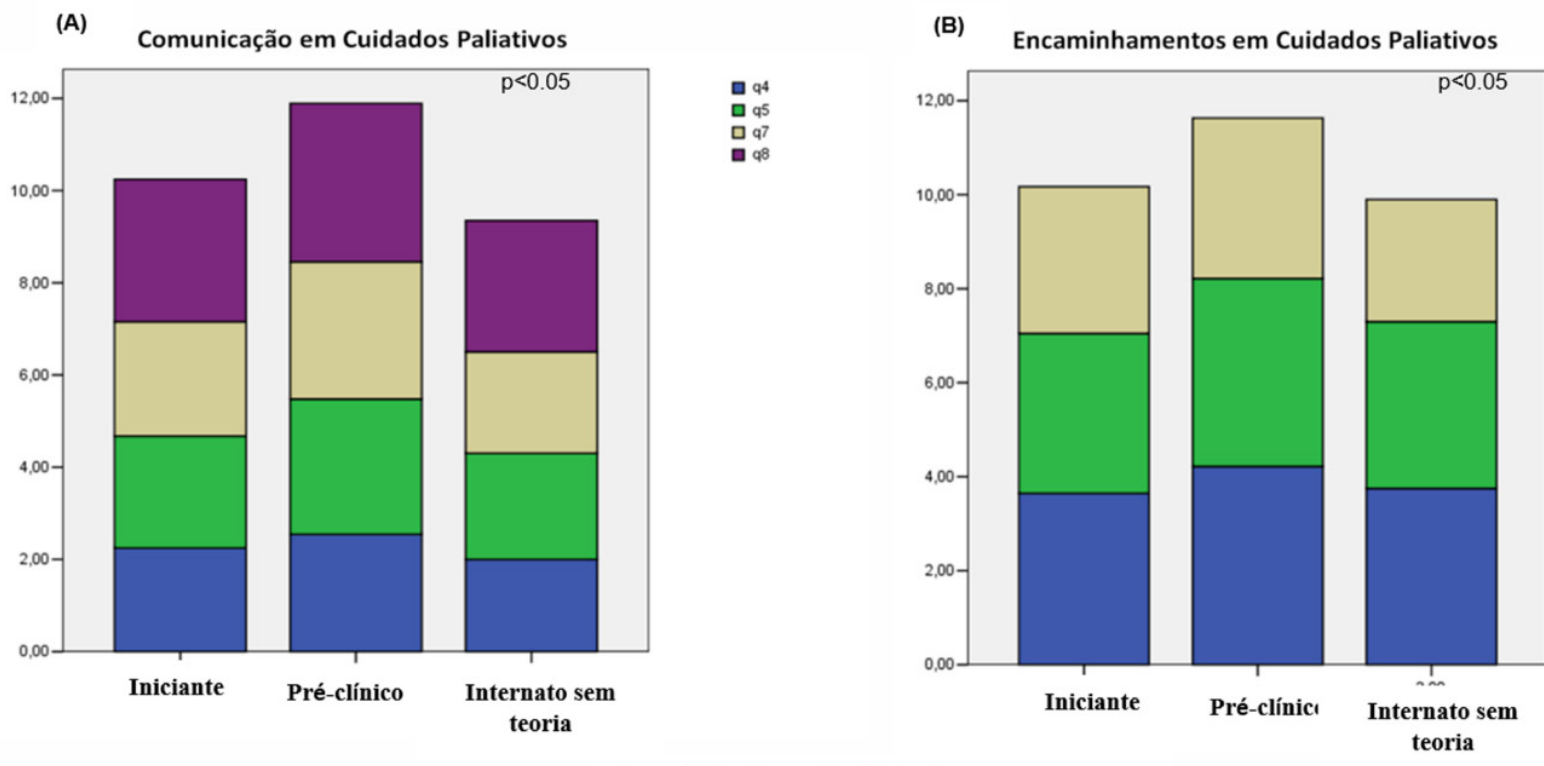

Kruscal-Wallis; $\mathrm{p}=$ significância 


\section{RESULTADOS}

O estudo do $n$ amostral mostrou serem suficientes 91 sujeitos de pesquisa. Foram observados 248 indivíduos, tendo sido excluídos oito. A casuística apresenta-se descrita na Tabela 1.

\begin{tabular}{|c|c|c|c|c|}
\hline $\begin{array}{r}\text { Casuística } \\
\text { sobre ati }\end{array}$ & $\begin{array}{l}\text { dos estudar } \\
\text { tudes e cor }\end{array}$ & $\begin{array}{r}\text { TAвELA } \\
\text { tes de Me } \\
\text { heciment }\end{array}$ & dicina de es & $\begin{array}{l}\text { udo de coorte } \\
\text { o paliativo }\end{array}$ \\
\hline Estudo & Gên & ero & I & ade \\
\hline & Masculino & Feminino & Faixa etária & № de pessoas \\
\hline Coorte & 42 & 52 & $19-25$ & 68 \\
\hline & & & $26-30$ & 12 \\
\hline & & & $31-35$ & 2 \\
\hline & & & $36-40$ & 1 \\
\hline & & & $>40$ & 1 \\
\hline Transversal & 47 & 76 & $19-25$ & 110 \\
\hline & & & $26-30$ & 13 \\
\hline & & & $31-35$ & 1 \\
\hline & & & $36-40$ & 0 \\
\hline & & & $>40$ & 1 \\
\hline
\end{tabular}

Fazendo-se uma análise pontual no momento da aplicação do questionário (M0), pôde-se observar o seguinte:

\section{Multidimensional Fear of Death Scale (MFODS)}

O MFODS avalia o medo da morte por meio de questões com situações relacionadas à morte. Ao final do teste, as questões são divididas em grupos de medo da morte - medo do processo de morrer, dos mortos, de ser destruído, medo por aqueles importantes para você, do desconhecido, da morte consciente, pelo corpo depois da morte e da morte prematura -, revelando em quais aspectos a morte gera maior ansiedade no indivíduo. Ao se agruparem as questões segundo o tipo de medo que cada uma representa, observa-se que o "medo por aqueles importantes para você" apresenta-se como o tipo predominante, seguido pelo "medo dos mortos" e "medo do desconhecido" (Figura 1).

As maiores diferenças foram vistas entre os grupos iniciante e pré-clínico, nas questões "Tocar em um cadáver não me incomodaria" (questão 14); "Como todos morrem, não ficarei tão chateado quando meus amigos morrerem" (questão 25); “Não importa se eu for enterrada em um caixão de madeira ou em um compartimento de aço" (questão 28); "Me assusta pensar que eu posso estar consciente enquanto estiver em um necrotério" (questão 29), sendo demonstrado maior medo pelo grupo pré-clínico quando avaliadas as questões 14 e 28. O grupo internato com prática sem teoria mostrou menor medo do que o grupo iniciante nas questões avaliadas acima, exceto quanto a "Tocar em um cadáver não me incomodaria" (questão 14). Na questão "Não quero doar meus olhos depois que eu morrer" (questão 33), o grupo internato com prática sem teoria mostrou menos medo do que o grupo pré-clínico. De modo geral, o grupo que apresenta menor medo da morte é o dos iniciantes.

\section{Self-Efficacy in Palliative Care Scale (SEPCS)}

O SEPCS apresenta sentenças relacionadas a assuntos e experiências comuns à unidade de cuidado paliativo. Elas se relacionam a comunicação, técnica específica e trabalho em equipe multidisciplinar. As respostas são utilizadas para avaliar o grau de ansiedade do indivíduo. O grupo pré-clínico mostrou-se mais confiante (Figura 2) que os demais, em especial quanto a "comunicação" em CP e "trabalho em equipe multidisciplinar", seguido pelo grupo iniciante. Pontos específicos deste questionário relacionados a "comunicação" em CP e "trabalho em equipe multidisciplinar" podem demonstrar que estudantes sem formação teórica e/ou sem prática em Cuidado Paliativo guardam maior ansiedade quanto às reflexões abordadas (Figura 3).

Ao se comparar o desenvolvimento dos estudantes ao longo do tempo (estudo de coorte), pôde-se observar o seguinte:

\section{Multidimensional Fear of Death Scale (MFDOS)}

O Multidimensional Fear of Death Scale (MFDOS) avalia o medo da morte por meio de questões com situações relacionadas à morte. Ao final do teste, as questões são divididas em grupos de medo da morte - medo do processo de morrer, dos mortos, de ser destruído, medo por aqueles importantes para você, do desconhecido, da morte consciente, pelo corpo depois da morte e da morte prematura -, revelando em quais aspectos a morte gera maior ansiedade no indivíduo. $\mathrm{O}$ grupo pré-clínico mostra menor ansiedade relacionada ao processo de morrer. Ao longo do segundo período de formação, do período pré-clínico ao internato observam-se duas mudanças significativas: aumento do medo de ser destruído e da morte prematura (Tabela 2). 


\begin{tabular}{|c|c|c|c|c|c|c|c|c|}
\hline $\begin{array}{r}\text { Evoluçâa } \\
\text { es }\end{array}$ & $\begin{array}{l}\text { ão ao longo do tempo do Multidimensi } \\
\text { tudantes iniciantes, em período pré-cli }\end{array}$ & $\begin{array}{l}\text { TABELA } 2 \\
\text { ional Fear of } I \\
\text { línico do curs }\end{array}$ & $\begin{array}{l}\text { Death Sca } \\
\text { o e no inte }\end{array}$ & $\begin{array}{l}\text { ale }(M F O D \\
\text { ternato, Bra }\end{array}$ & $\begin{array}{l}\text { S) con } \\
\text { agança }\end{array}$ & $\begin{array}{l}\text { nforme os } \\
\text { a Paulista, }\end{array}$ & $\begin{array}{l}\text { grupos de } \\
2016\end{array}$ & \\
\hline & & & Evo & blução & & & rolução & \\
\hline Tipo de medo & Questão & № da questão & Iniciante & Pré-clínico & $\mathrm{p}$ & Pré-clínico & $\begin{array}{l}\text { Internato com } \\
\text { prática e teoria }\end{array}$ & p \\
\hline Processo de morrer & Tenho medo de morrer lentamente & 1 & 2,00 & 3,13 & $0,00^{*}$ & 2,00 & 1,61 & 0,08 \\
\hline De ser destruído & Gostaria de doar meu corpo para a ciência & 3 & 4,21 & 3,80 & 0,22 & 4,17 & 2,79 & $0,00^{*}$ \\
\hline $\begin{array}{l}\text { Por aqueles } \\
\text { importantes }\end{array}$ & $\begin{array}{l}\text { Tenho medo da morte de pessoas da } \\
\text { minha família }\end{array}$ & 4 & 1,18 & 2,00 & $0,00^{*}$ & 1,73 & 1,61 & 0,59 \\
\hline para você & $\begin{array}{l}\text { Se as pessoas das quais eu sou muito } \\
\text { próximo estivessem para morrer em breve, } \\
\text { eu sofreria por muito tempo }\end{array}$ & 16 & 1,21 & 2,26 & $0,00^{*}$ & 1,97 & 2,09 & 0,59 \\
\hline & $\begin{array}{l}\text { Se estivesse para morrer amanhã, minha } \\
\text { família ficaria triste por muito tempo }\end{array}$ & 17 & 1,34 & 2,13 & $0,01^{*}$ & 1,71 & 1,72 & 0,97 \\
\hline & $\begin{array}{l}\text { Como todos morrem, não ficarei tão } \\
\text { chateado }\end{array}$ & 25 & 1,53 & 2,60 & $0,01^{*}$ & 1,93 & 2,05 & 0,64 \\
\hline Do desconhecido & $\begin{array}{l}\text { Ninguém pode dizer, com certeza, o que } \\
\text { acontece depois da morte }\end{array}$ & 36 & 1,43 & 2,26 & $0,02^{*}$ & 2,47 & 2,18 & 0,36 \\
\hline Dos mortos & Tenho medo de coisas que já morreram & 39 & 4,21 & 2,86 & $0,00^{*}$ & 3,76 & 3,75 & 0,99 \\
\hline $\begin{array}{l}\text { Da morte } \\
\text { prematura }\end{array}$ & $\begin{array}{l}\text { Tenho medo de não viver o bastante para } \\
\text { aproveitar minha aposentadoria }\end{array}$ & 12 & 3,09 & 2,86 & 0,65 & 3,56 & 2,83 & $0,00^{*}$ \\
\hline
\end{tabular}

\section{TABELA 3}

Evolução do Self-Efficacy in Palliative Care Scale (SEPCS) relacionada a "comunicação" em Cuidados Paliativos e "trabalho em equipe multidisciplinar", avaliando os grupos de estudantes iniciantes e depois em período pré-clínico e do pré-clínico ao internato, Bragança Paulista, 2016

\begin{tabular}{|c|c|c|c|c|c|c|c|}
\hline \multirow[t]{2}{*}{ Habilidade } & \multirow[t]{2}{*}{ Questão } & \multicolumn{2}{|c|}{$\begin{array}{l}\text { Evolução da ausência de } \\
\text { formação específica para } \\
\text { aquisição do conhecimento } \\
\text { teórico (média das } \\
\text { questões corretas) }\end{array}$} & \multicolumn{4}{|c|}{$\begin{array}{l}\text { Evolução do conhecimento } \\
\text { teórico ao conhecimento } \\
\text { teórico e prático (média } \\
\text { das questões corretas) }\end{array}$} \\
\hline & & $\begin{array}{l}\text { Iniciante } \\
\text { (média das } \\
\text { questões } \\
\text { corretas) }\end{array}$ & $\begin{array}{l}\text { Pré-clínico } \\
\text { (média das } \\
\text { questões } \\
\text { corretas) }\end{array}$ & $\mathrm{p}$ & Pré-clínico & $\begin{array}{l}\text { Internato } \\
\text { com prática } \\
\text { e teoria }\end{array}$ & $\mathrm{p}$ \\
\hline \multirow[t]{3}{*}{ Comunicação } & $\begin{array}{l}\text { Discutir os prováveis efeitos do câncer com o } \\
\text { paciente }\end{array}$ & 1 & 2,00 & 0,00 & 2,00 & 3,19 & 0,00 \\
\hline & $\begin{array}{l}\text { Discutir a morte do paciente (que está por vir) } \\
\text { com sua família }\end{array}$ & 0 & 2,54 & 0,00 & 2,54 & 3,15 & 0,00 \\
\hline & $\begin{array}{l}\text { Discutir a morte do paciente com a família com } \\
\text { relação à perda/privação }\end{array}$ & 0 & 2,93 & 0,00 & 2,93 & 3,36 & 0,04 \\
\hline \multirow{3}{*}{$\begin{array}{l}\text { Trabalho } \\
\text { em equipe } \\
\text { multiprofissional }\end{array}$} & $\begin{array}{l}\text { Quanto a trabalhar com uma equipe } \\
\text { multiprofissional em Cuidados Paliativos }\end{array}$ & 0 & 3,30 & 0,00 & 3,30 & 3,87 & 0,01 \\
\hline & $\begin{array}{l}\text { Quanto a encaminhar corretamente pacientes } \\
\text { paliativos para serviço de linfoedema (profilaxia } \\
\text { de edema linfático devido a esvaziamento } \\
\text { linfonodal) }\end{array}$ & 0 & 4,00 & 0,00 & 4,00 & 3,51 & 0,03 \\
\hline & $\begin{array}{l}\text { Quanto a encaminhar corretamente pacientes } \\
\text { paliativos para avaliação psiquiátrica }\end{array}$ & 0 & 3,41 & 0,00 & 3,41 & 4,24 & 0,00 \\
\hline
\end{tabular}




\section{Self-Efficacy in Palliative Care Scale (SEPCS)}

Pontos específicos do questionário SEPCS relacionados a "comunicação" em Cuidados Paliativos e "trabalho em equipe multidisciplinar" demonstraram a melhora das habilidades e da ansiedade relacionadas à associação da teórica à prática ao longo da formação médica. Observou-se que, ao chegarem à fase pré-clínica, os estudantes apresentam menos medo quanto à comunicação (questão 1). A grande diferença se concentra no período entre a formação pré-clínica e o internato, onde, exceto pela questão sobre o encaminhamento correto de pacientes paliativos, os acadêmicos no decurso do internato que tiveram formação teórica em CP obtiveram desempenho superior (Tabela 3).

\section{DISCUSSÃO}

O medo da morte está relacionado a fatores emocionais, cognitivos e motivacionais que variam de acordo com o contexto socioeconômico, cultural e pessoal de cada indivíduo ${ }^{20}$ e por isso é tido como "multidimensional".

Para mensuração da influência dos estudos em cuidados de final de vida na formação médica, a união de questionários que avaliam as esferas cognitiva e afetiva em intervalo temporal ao longo da formação médica permite uma análise que vai além da simples mudança de atitude do estudante de Medicina, trazendo uma visão clara da articulação na transição de conhecimentos e habilidades adquiridos para mudança de comportamento. Esta observação contradiz a hipótese apresentada por pesquisador que sugere que este tipo de mensuração não seria possível ${ }^{21}$.

$\mathrm{O}$ intuito da disciplina de CP é, ao colocar o estudante em diversas situações de conflito do binômio "vida e morte", fazer com que ele desenvolva suas ferramentas cognitivas e afetivas, para que, ao se deparar com a situação real, não tenha um impacto que o faça sofrer de maneira disfuncional. Dessa forma, o grupo "exposto" à disciplina seria representado por cuidadores mais seguros de suas atitudes, como afirma Esslinger ${ }^{15}$. Como esperado, no presente estudo, as diferenças se situam no desenvolvimento das habilidades cognitivas e afetivas entre estudantes iniciantes e aqueles expostos à teoria e à realidade prática associada a fundamentos teóricos embasados, corroborando relatos da literatura ${ }^{14}$.

De forma inesperada, no presente estudo, os iniciantes pouco se distanciam dos internos que não tiveram contato com a teoria e apenas foram expostos, no treinamento em serviço, às situações práticas de terminalidade de vida, contrapondo-se à hipótese de Sullivan ${ }^{21}$. Aqueles têm conhecimento sobre a morte decorrente de experiências pessoais e se deparam com este tema, na academia, apenas quando em contato com cadá- veres. Apesar da ausência do conhecimento técnico, os alunos iniciantes não demonstram resultados tão díspares quanto ao medo da morte em relação ao grupo que se encontra no internato, para o qual poderá a morte ser personificada como "fracasso". O grupo que está no internato desenvolve convívio diário com a doença e com a morte sem elaborar, na teoria, a possibilidade da perda do paciente, mas acaba por se tornar capaz de desenvolver suas próprias ferramentas cognitivas / afetivas para suportar as exposições diárias à morte, à custa de maior ansiedade do que a dos estudantes que tiveram a chance de elaborar as questões relacionadas, ainda que apenas no contexto teórico. Tal observação parece indicar a condução pessoal e empírica dos internos nesta fase e não a condução técnica esperada. Aqueles expostos ao aprendizado em serviço apresentam semelhanças com o grupo de estudantes que nunca foi exposto à questão da perda advinda da morte, o que confirma o impacto que tem a morte nos anos finais de formação do médico, como se ele fosse tão leigo quanto a população em geral ou aquela que acaba de iniciar seus estudos.

Estudantes que tiveram formação teórica em $\mathrm{CP}$, mas não se iniciaram na prática apresentam menor ansiedade diante da morte, com melhor desempenho do que os iniciantes, porém são mais ansiosos com relação à figura do cadáver. Para quem ingressa na faculdade, o cadáver representa uma fonte de conhecimentos fundamental a sua formação médica, além de estar presente no cotidiano devido à carga de morfologia e anatomia que compõe grande parte da grade horária, o que justifica este achado. Para os alunos do ciclo pré-clínico, o cadáver pode estar associado à morte, sendo então o que gostaria de evitar: perder um doente, ainda que hipoteticamente, no futuro, o "fracasso". Os internos que receberam formação teórica e prática em CP são aqueles que apresentam menor medo dos mortos e da morte, demonstrando que a ansiedade relacionada a este medo pode ser reduzida com a teoria e a prática, corroborando dados de autor que afirma que "a vivência prática é absolutamente necessária, pois contextualiza a aprendizagem e sedimenta a nova atitude"22.

Comparando-se o ponto específico relacionado à doação de órgãos, de importância fundamental para o estabelecimento de novas regras e a promoção desta necessidade cada vez mais importante na saúde mundial, o grupo que discute a teoria apresenta melhor desempenho do que o interno ao qual não foi oferecido contato prévio com a reflexão teórica. Naqueles que têm formação teórica e prática em CP, observa-se maior confiança quanto à finitude da vida e, desta forma, enquanto futuros profissionais da saúde, poderão desempenhar melhor sua atividade nos grupos de doação de órgãos nos serviços aos quais irão se integrar. 
Da mesma forma, as habilidades em comunicação e interdisciplinaridade em CP são mais bem desempenhadas pelo grupo que recebeu treinamento teórico e prático. Isto pode ser explicado pela teoria de Auto-Eficácia de Bandura (1986), que define autoeficácia como "a crença que o indivíduo tem sobre sua capacidade de realizar com sucesso determinada atividade. Dessa forma, sua crença pode afetar suas escolhas e o desempenho profissional." ${ }^{23}$ (p. 1). Trata-se de avaliação pessoal das próprias habilidades, não apenas quanto a tê-las ou não, mas, sobretudo, em acreditar que as tem. Portanto, a teoria aliada à prática é o que de fato faz o aluno acreditar que possui tal habilidade.

A análise de autoeficácia demonstra claramente o quanto a ansiedade com relação à morte afeta o comportamento dos futuros médicos. Aqueles que refletiram sobre a morte e assuntos relacionados são os mesmos que se mostram mais confiantes para se comunicar com o paciente e sua família, controlar sintomas que geram mais desconforto ao paciente grave e até mesmo inserir o paciente no contexto de acompanhamento multiprofissional, como descrito por Billings em $1997^{24}$. Curiosamente, os estudantes iniciantes não se mostram muito diferentes do grupo de internos sem teoria em cuidados paliativos quanto à ansiedade relacionada às habilidades de comunicação e condutas em CP. Esta insegurança é uma tendência normal observada entre estudantes de Medicina antes de passarem pela vivência teórica e prática, reafirmando a necessidade da vivência teórica anteriormente à prática, corroborando dados da literatura ${ }^{14}$.

Quanto à comunicação, mais especificamente a comunicação de más notícias, o grupo de internos sem exposição à teoria demonstra menor confiança na habilidade para se comunicar com o paciente e sua família, apesar de demonstrar conhecer as condutas adequadas, denunciando, mais uma vez, a insegurança que cerca estes estudantes. Este dado corrobora achado de Gruber ${ }^{25}$, que demonstra que, com o passar dos anos de formação médica, há tendência de omissão de dados que dizem respeito a condutas, tomada de decisões e até desfechos iatrogênicos na relação com o paciente.

Resultado semelhante foi observado em estudo que verificou que enfermeiras com maior grau de ansiedade se mostraram mais relutantes em discutir assuntos relacionados à mor$t^{17}$. Esta é, sem dúvida, uma observação preocupante e, uma vez mais, demonstra a contribuição dos CP na elaboração desta questão, visto que incentiva a comunicação como principal ferramenta para a construção de uma relação médico-paciente satisfatória, fato ressaltado por outros autores ${ }^{14}$. Também se observa a dificuldade de trabalhar em equipe e de compreender a saúde e os CP como obrigação multidisciplinar naqueles estudantes com formação parcial.
Ao longo da formação médica, o medo da morte, entretanto, não é superado apenas pelo contato teórico. A morte representa a ineficácia do tratamento proposto, gerando, portanto, frustração no estudante que ainda não consegue elaborar o medo de forma completa. O contato teórico melhora a superação de medos específicos, como aquele derivado do processo da própria morte ou da perda de pessoas importantes. $\mathrm{O}$ fato de os estudantes aprenderem maneiras de controle da dor, comunicação e atendimento completo do paciente grave torna o processo de morrer mais humanizado e mais aceitável para si e para aqueles a sua volta.

Pode-se observar, ao longo do tempo, a melhora quanto ao "medo do desconhecido", o que demonstra que a discussão reduz a ansiedade quanto ao que vem após a morte. Um estudo de Filho e colaboradores ${ }^{26}$ discute a necessidade e as dificuldades de introduzir cursos relacionados à espiritualidade na formação médica. Esta dificuldade decorre da ausência de estudos nacionais sobre o assunto e do preconceito existente contra a coerção e o sectarismo religioso. Há dificuldade dos estudantes em diferenciar espiritualidade e religião, além do receio de, ao lidarem com o tema, se afastarem da "medicina real". Apesar de tudo, constatou-se mudança na maneira como os acadêmicos percebem o assunto e como este influencia positivamente sua relação com a morte.

Os internos com formação teórica e prática em CP apresentaram maior medo da morte prematura e de serem destruídos. Isto pode estar relacionado às discussões efetuadas sobre o processo de morrer e, ainda que de forma subconsciente, ao reconhecimento da responsabilidade da indução de mistanásia ou distanásia na atuação profissional futura. Tendo-se mais conhecimento de como algumas doenças podem ser lentamente fatais, adquire-se mais medo da morte, não pelo seu fim, mas pelo seu meio, especialmente com o desenvolvimento tecnológico médico alcançado nos dias atuais. Bendassolli, em $2001^{27}$, em estudo que relaciona doação de órgão e medo da morte, revela que, entre os indivíduos entrevistados, também estudantes de Medicina, a maioria dos que se mostraram contrários à doação de órgãos apresentava receio da morte prematura ou premeditada em prol da doação de órgãos - ou seja, adiantar a morte com a finalidade de comercialização de órgão - , de forma distinta do encontrado no presente estudo, onde, embora os acadêmicos com formação teórica e prática tenham demonstrado medo da morte prematura, estão mais bem preparados para a comunicação em doação de órgãos.

Quanto à avaliação de autoeficácia em CP, a formação completa gera melhor desempenho em relação à comunicação de más notícias. O grupo do internato com formação completa em CP teve importante incremento não apenas da habilidade 
de comunicação, como também do trabalho interdisciplinar, discordando de Sullivan et al. ${ }^{21}$, que afirmaram que, nos dias de hoje, os esforços despendidos para introduzir o ensino em terminalidade de vida nas escolas de Medicina não se traduziram em melhorias significativas na educação médica. No presente estudo, demonstrou-se o quanto o contato teórico reflexivo é modificador, em especial na questão da comunicação, grande chave da relação médico-paciente, que permite aplicar os cuidados e tranquilizar a família e o paciente, sendo este o cuidar verdadeiramente eficaz. A multidisciplinaridade representa a medicina centrada não no médico, mas na equipe que cuida e deixa ser cuidada. Aqueles a quem não foi oferecido refletir durante a disciplina teórica demonstram saber como agir perante o paciente paliativo, porém são mais inseguros em relação às condutas e aos próprios receios quanto à morte, gerando ansiedade desnecessária. Como consequência, pode-se aumentar o risco da Síndrome de Burnout, cujo desencadeador é o desequilíbrio entre ansiedade e resolutividade, medo e insegurança, além da carga de trabalho e radiações psicológicas sofridas.

É importante ressaltar que as condições deste estudo foram bastante específicas e ocorreram diante de uma mudança curricular, o que poderá ter influenciado os resultados, e que estratégias de ensino diversas podem gerar resultados semelhantes, sendo necessários outros estudos para que a generalização possa ser considerada em sua plenitude.

Pode-se afirmar que o ensino em CP aplicado em disciplina com foco primário e o desenrolar reflexivo associado ao ensino prático fornecem ao futuro médico um alicerce para conduzir adequadamente pacientes graves quanto aos cuidados de fim da vida. Concordam com estes resultados Fortes e colaboradores $^{28}$, que demonstraram que médicos que tiveram aprendizado em cuidados de fim de vida e leram artigos relacionados ao tema apresentam maior adesão e aceitação em aplicar CP de forma correta e sem ansiedade para si e para seus pacientes. Portanto, a educação médica, preferencialmente ainda no período da graduação, é crucial para que a prática dos CP seja aceita no dia a dia profissional e representa uma ferramenta importante para moldar a postura dos médicos perante a morte e o processo de morrer. Atividades educacionais que proporcionem ao futuro médico momentos de reflexão e ganho de perspectiva são estratégias fundamentais para se compreender o humanismo e a empatia, características inerentes ao verdadeiro profissional médico ${ }^{29}$.

O método de ensino, a simulação de situações reais, a interdisciplinaridade com professores de outras áreas, a forma de avaliação do conteúdo e o cuidado em discutir temas como ética e espiritualidade antes do início da prática em serviço e no internato estão incluídos no que é tido como o melhor mé- todo de ensino em CP, em acordo com os nossos resultados e com o que preconizam Pessini e Bertachini22 ${ }^{2}$ O ensino em CP com desenrolar reflexivo fornecerá alicerce à condução apropriada dos pacientes nos cuidados de fim da vida.

Pode-se concluir, com base nos resultados do presente estudo, que a educação médica em $\mathrm{CP}$, de forma integral, teórica e prática, é crucial para a prática da Medicina e representa uma ferramenta importante para moldar a postura dos médicos perante a morte e o processo de morrer.

\section{REFERÊNCIAS}

1. Silva, EP, Sudigursky, D. Concepções sobre cuidados paliativos: revisão bibliográfica. Acta Paul Enferm 2008;21(3): 504-8.

2. World Health Organization. Pain Relief and Palliative Care. 2a ed. Geneva: WHO; 2002.

3. Conselho Federal de Medicina. Código de Ética Médica. Resolução CFM no 1931, de 17 de setembro de 2009. Aprova o Código de Ética Médica. Diário Oficial da União, 24 set. 2009. Seção I, p. 90-2. Disponível emwww.in.gov.br.

4. Conselho Regional de Medicina do Estado de São Paulo. Medicina Paliativa é oficializada como área de atuação diz Jornal do CREMESP [online]. 2010, p.6-7 [acesso em 20 de março 2010]. Disponível em www.cremesp.org.br

5. Dickinson GE. Death education in U.S. medical schools: 1975-1980. Med Educ 1981;2(56):111-14.

6. Barzansky B, Veloski JJ, Miller R, Jonas HS. Education in end-of-life care during medical school and residency training. Academic Medicine 1999;10(74):102-104.

7. Head BA, Schapmire TJ, Earnshaw L, et al. Improving medical graduates' training in palliative care: advancing education and practice. Advances in Medical Education and Practice. 2016;7:99-113. doi:10.2147 / AMEP.S94550.)

8. Toledo, AP; Priolli, DG. Cuidados no fim da vida: o ensino médico no Brasil. RevBrasEducMed 2012;36(1):109-117.

9. Oliveira RA, coord. Cuidado Paliativo. São Paulo: Conselho Regional de Medicina do Estado de São Paulo; 2008.

10. Head, BA, Schapmire, TJ, Shaw, MA. Improving medical graduates' palliative care: advancing education and practice. Adv Med EducPract. 2016;7:99-113.

11. Support Principal Investigators. A controlled trial to improve care for seriously ill hospitalized pacients. Study to Undestand Prognoses and Preferences for Outcomes and Risks of Treatment (SUPPORT). The SUPPORT Principal Investigators. JAMA 1995;274(20):1591-8.

12. Pype P; Symons L; Wens J; Van den Eynden B; Stes A; Deveugele $M$. Health care professionals' perceptions towards lifelong learning in palliative care for general practitioners: a focus group study. BMC FamPract2014;15:36. 
13. Bifulco, VA, Iochida, LC. A formação na graduação dos profissionais de saúde e a educação para o cuidado de pacientes fora de recursos terapêuticos de cura. Rev Bras Educ Med2009;33(1):92-100.

14. Mason SR, Ellershaw JE. Preparing for palliative medicine; evaluation of an education programme for fourth year medical undergraduates. J PalliatMed 2008;22(6):687-92.

15. Esslinger, I. De quem é a vida, afinal?- Descortinando os cenários da morte. 1ª edição. São Paulo, Ed. Loyol; 2004. p.216-220.

16. Buckman, R. How to Break Bad News: a Guide for Health Care Professions. Baltimore, MD: the John Hopkins University Press; 1992. p.65-97.

17. Neimeyer, R.A.; Moore, M.K. Validity and reliability of the multidimensional fear of death scale. In R. A. Neimeyer (Ed.), Death anxiety handbook: Research, Instrumentation and Application. Washington, DC: Taylor \& Francis;1994. p.103-117.

18. Mason, SR, Ellershaw, JE. Undergraduate training in palliative medicine: is more necessarily better? J PalliatMed 2010;24(3):306-309.

19. Santos, Glauber Eduardo de Oliveira. Cálculo amostral: calculadora on-line. Disponível em: <http://www.calculoamostral.vai.la>. Acesso em: [08 de abril de 2012].

20. Letho, R.H., Stein, K.F. Death Anxiety: An Analysis of an Envolving Concept. Research and Theory for Nursing Practice: An Int Journal 2009;23(1):23-41.

21. Sullivan AM, Warren AG, Lakoma MD, Liaw KR, Hwang D, Block SD. End-of-life care in the Curriculum: A National Study of Medical Education Deans. AcadMed 2004;79(8):760-8.

22. Pessini, L, Bertachini, L. Novas perspectivas em cuidados paliativos: ética, geriatria, gerontologia, comunicação e espiritualidade. Mundo Saúde 2005;29(4):491-509.

23. Barros M, Santos, ACB. Por dentro da autoeficácia: um estudo sobre seus fundamentos teóricos, suas fontes e conceitos correlatos. Revista Espaço Acadêmico, no ${ }^{\text {112. Set, }}$ 2010, p.1-9. http://eduem.uem.br/ojs/index.php/EspacoAcademico/article/viewFile/10818/5961
24. Biliings, M.E., Engelberg, R., Curtis, J.R., Block, S., Sullivan, A.M. Determinants of medical students' perceived preparation to perform end-of-life care, quality of end-of-life care education, and attitudes towards end-of-life care. J PalliaT Med 2010;13(3): 319-326.

25. Gruber PC, Gomersall CD, Joynt GM, Lee A, Tang PY, Young AS, Yu NY, Yu OT. Changes in medical students' attitudes towards end-of-life decisions across different years of medical training. J GenInternMed 2008; 23(10):1608-14.

26. Filho JCG, Beraldi, GH, Nunes, MPT, Gannam, S. Espiritualidade no Curriculum das Escolas Médicas no Mundo e no Brasil. Revista de Educação Permanente em Saúde; 2010;34:136.

27. Bendassoli, PF. Percepção do Corpo, Medo da Morte, Religião e Doação de Órgãos. Psicologia: Reflexão e Crítica 2001;14(1):225-240.

28. Fortes, D.N., Vincent, J.L., Velasco, I.T., Park, M. Association between education in EOL care and variability in EOL practice: a survey of ICU physicians. Intensive Care Med 2012;38(3):404-412.

29. Braun, U.K., Gil, A.C., Teal, C.R., Morrison, L.J. The Utility of Reflective Writing after a Palliative Care Experience: Can We Assess Medical Students' Professionalism? Palliat Med 2013;16(11):1342-1349.

\section{CONTRIBUIÇÃO DOS AUTORES:}

Todos os autores contribuiram em todas as fases, analisando e escrevendo os artigos.

\section{CONFLITO DE INTERESSES:}

Os autores declaram ausência de conflito de interesse

\section{ENDEREÇO PARA CORRESPONDÊNCIA}

Denise Gonçalves Priolli

Rua São Vicente, 614

Jardim Paulista, Atibaia- São Paulo, Brasil

CEP 12947-390

Tel: 551124548298 